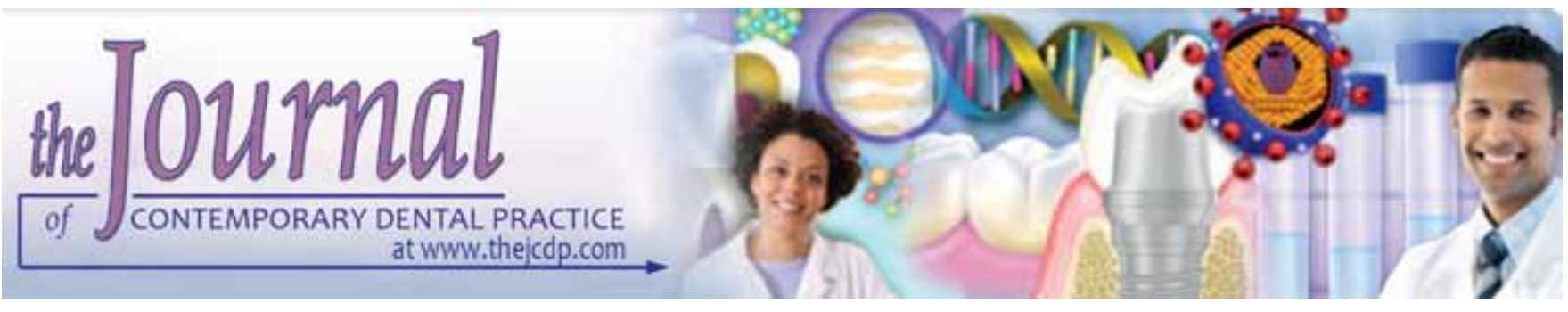

\title{
Direct Measurement of Stain Retention in Third Molars
}

\author{
R Steven Kurti Jr, Berj Der Boghossian, So Ran Kwon
}

\section{ABSTRACT}

Aim: To directly determine the mass of dye retained in teeth following exposure to aqueous solutions of Rhodamine B and to correlate tooth color modifications.

Materials and methods: Extracted third molars (25) were selected and sectioned at the cementoenamel junction for coronal staining. Pulp tissue was removed and teeth sonicated to remove debris. Teeth were kept in deionized water for 12 hours and subsequently weighed. They were then stained for 4 hours in $5 \mathrm{ml}$ of Rhodamine $B$ dye at two different concentrations. The samples were then subjected to two 8 hours rinses in deionized water. The tooth shade was recorded with a commercially available intraoral spectrophotometer (Vita Easyshade Compact, Vita Zahnfabrik, Bad Säckingen, Germany) at baseline ( $\left.T_{1}\right)$, after dye immersion $\left(T_{2}\right)$, and after water rinsing $\left(T_{3}\right)$. A standard absorption curve was then used to calculate the dye mass in the rinse solutions as well as the posttreatment stain solutions. All solution optical absorption curves were recorded using a laboratory research spectrophotometer (Cary 300 , Agilent, USA). The mass of dye in each solution was then calculated from the standard curve relating optical absorption to aqueous dye concentration.

Results: An average change in the CIE (a) values of $8.0 \pm 0.3$ were observed for concentrations of Rhodamine B similar to the optical appearance of wine or other darkly colored juices while an increase of $10 x$ in concentration gave values too high to measure using a standard intraoral spectrophotometer. By measuring the optical absorbance of the staining solutions before and after the staining process, we were able to measure dye retention of $54 \pm 26$ micrograms per gram of tooth.

Conclusion: While no significant correlation could be found between the amount of stain retention in the dentition and the tooth shade due to the high uncertainties in the spectroscopic measurements, we were able to show that this method should admit such comparisons for future research.

Clinical significance: The development of a reliable chromophore infiltration model may provide standardized and reproducible results in evaluating tooth whitening efficacy.

Keywords: Tooth whitening, Chromophore, Color change, Absorption spectrum, Dye retention.

How to cite this article: Kurtir Jr RS, Boghossian BD, Kwon SR. Direct Measurement of Stain Retention in Third Molars. J Contemp Dent Pract 2013;14(6):1060-1064.

\section{Source of support: Nil}

Conflict of interest: None declared

\section{INTRODUCTION}

Tooth whitening is a noninvasive and highly effective method to whiten discolored teeth. It is a treatment option to enhance the esthetics of teeth that has been practiced in dentistry for over 100 years. Thus, the safety and efficacy of this procedure has been well established. ${ }^{1}$ However, the basic mechanism of tooth whitening with hydrogen peroxide as the main active ingredient has not been fully explained. Two main theories have been proposed which may both contribute to an increase in lightness and decrease in chroma in the yellow-blue axis observed after tooth whitening. The chromophore theory suggests that whitening works by oxidation of polymeric organic substances. Oxygen and hydroxyl radicals released from hydrogen peroxide are attracted to the complex ring structure with conjugated double bonds that exhibit electron-rich areas. Saturation or cleavage into more simple chain structures may lead to removal of the stain molecule or result in alteration the reflective index of the tooth, so that it appears lighter. ${ }^{2}$ The optical theory states that peroxides cause slight morphologic alterations in the enamel thus reducing its translucency by scattering light near the tooth surface. The increased opaqueness due to scattering masks the darker subjacent dentin layer affecting tooth color. ${ }^{3-5}$ In order to validate theories pertaining to whitening mechanism, there is a need for a model to evaluate stain penetration and stain retention in the tooth. Therefore the purpose of this study was to develop a model to determine the amount of dye retained in teeth following prolonged exposure to stain molecules and correlate stain retention to tooth color changes. The null hypothesis to be tested was that by using solution density and optical absorption spectrums it will be possible to calculate the mass of dye retained in extracted third molars after artificial chromophore infiltration. 


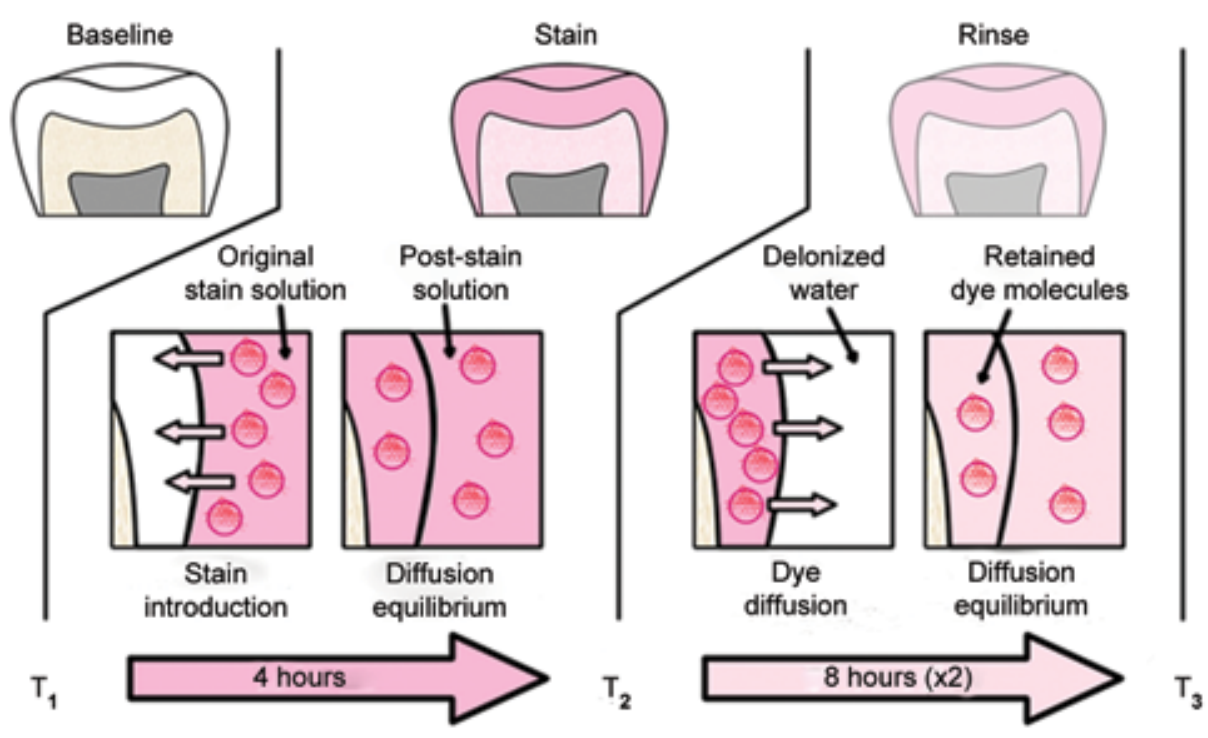

Fig. 1: Chromophore infiltration and rinse process

\section{MATERIALS AND METHODS}

\section{Sample Selection and Preparation}

Sound extracted human third molars (25) were collected prior to the study and stored in $0.1 \%$ thymol solution at $4{ }^{\circ} \mathrm{C}$. All teeth were cleaned and observed for the absence of developmental anomalies, caries, existing restorations and deep crack lines. The roots were sectioned at the cementoenamel junction and remaining pulp tissue removed at the coronal section.

\section{Tooth Color Measurements}

Teeth were measured for chromatic properties at the middle third of the buccal surface using an intraoral spectrophotometer (Vita Easyshade Compact, Vita Zahnfabrik, Bad Säckingen, Germany). Measurements were performed at baseline $\left(\mathrm{T}_{1}\right)$, after chromophore infiltration $\left(\mathrm{T}_{2}\right)$, and after rinsing $\left(\mathrm{T}_{3}\right)$ in a color controlled illumination box (MM 4e GTI Mini Matcher, GTI Graphic Technology, Inc, Newburgh, NY, USA) at CIE $\mathrm{D}_{65}$, a color temperature of $6500 \mathrm{~K}$ and light intensity of $\approx 1200$ lux.

\section{Chromophore Infiltration and Measurement of Dye Retention}

The chromophore infiltration and rinsing process is illustrated in Figure 1. Specimens were subjected to staining

\begin{tabular}{|c|c|c|}
\hline Color change parameter & Mean (SD) & Error (\%) \\
\hline Lightness $(\Delta \mathrm{L})$ & $-3.1(0.7)$ & 20 \\
\hline Chroma $(\Delta \mathrm{C})$ & $-1.3(0.9)$ & 70 \\
\hline Hue $(\Delta h)$ & $-17(0.7)$ & 4 \\
\hline Red/Green Balance $(\Delta \mathrm{a})$ & $8.0(0.4)$ & 4 \\
\hline Yellow/Blue Balance $(\Delta \mathrm{b})$ & $-2.3(0.9)$ & 40 \\
\hline
\end{tabular}

for 4 hours in a Rhodamine B solution $\left(1.50 \times 10^{-3} \mathrm{~g} / \mathrm{ml}\right)$, which leaves a bright pink hue (Fig. 2). On completion of color measurement after chromophore infiltration $\left(\mathrm{T}_{2}\right)$ each specimen was subsequently rinsed in $5 \mathrm{ml}$ of deionized water for 8 hours to allow stain molecules in the tooth structure to diffuse into the water. The solutions used for staining were measured before and after staining with a laboratory spectrophotometer (Cary 300, Agilent, USA), and a standard curve was used to calculate the concentration of dye in the solutions (pre- and post-stain as well as the rinse solutions) from each tooth (Figs 3 and Graph 1). Specimens received a second rinse in $5 \mathrm{ml}$ of water for 8 hours and a spectrum taken of the second rinse solution. After the second water rinse, specimens were measured for CIELAB chromatic properties using the intraoral spectrophotometer $\left(\mathrm{T}_{3}\right)$.

\section{RESULTS}

\section{Tooth Color Measurement}

The first set of data used a Rhodamine B concentration, which stained the teeth beyond the range that the intraoral spectrophotometer would measure (see Figure 2 for representative photos of teeth at $T_{1}, T_{2}$ and $T_{3}$ ). As a result a smaller set with a lower Rhodamine B concentration was obtained and color change data summarized in Table 1.

The overall reduction in lightness $(\Delta \mathrm{L})$ is due to the enhanced absorption of the Rhodamine $\mathrm{B}$ dye. The slight change in chroma $(\Delta \mathrm{C})$ is not particularly significant (note $70 \%$ error), but the change in hue $(\Delta \mathrm{h})$ is quite significant (note only $4 \%$ error in values). Similarly we do not see a particularly significant change in the blue/yellow color balance $(\Delta b)$, while there was a significant change in the $\mathrm{red} /$ green color balance $(\Delta \mathrm{a})$. 


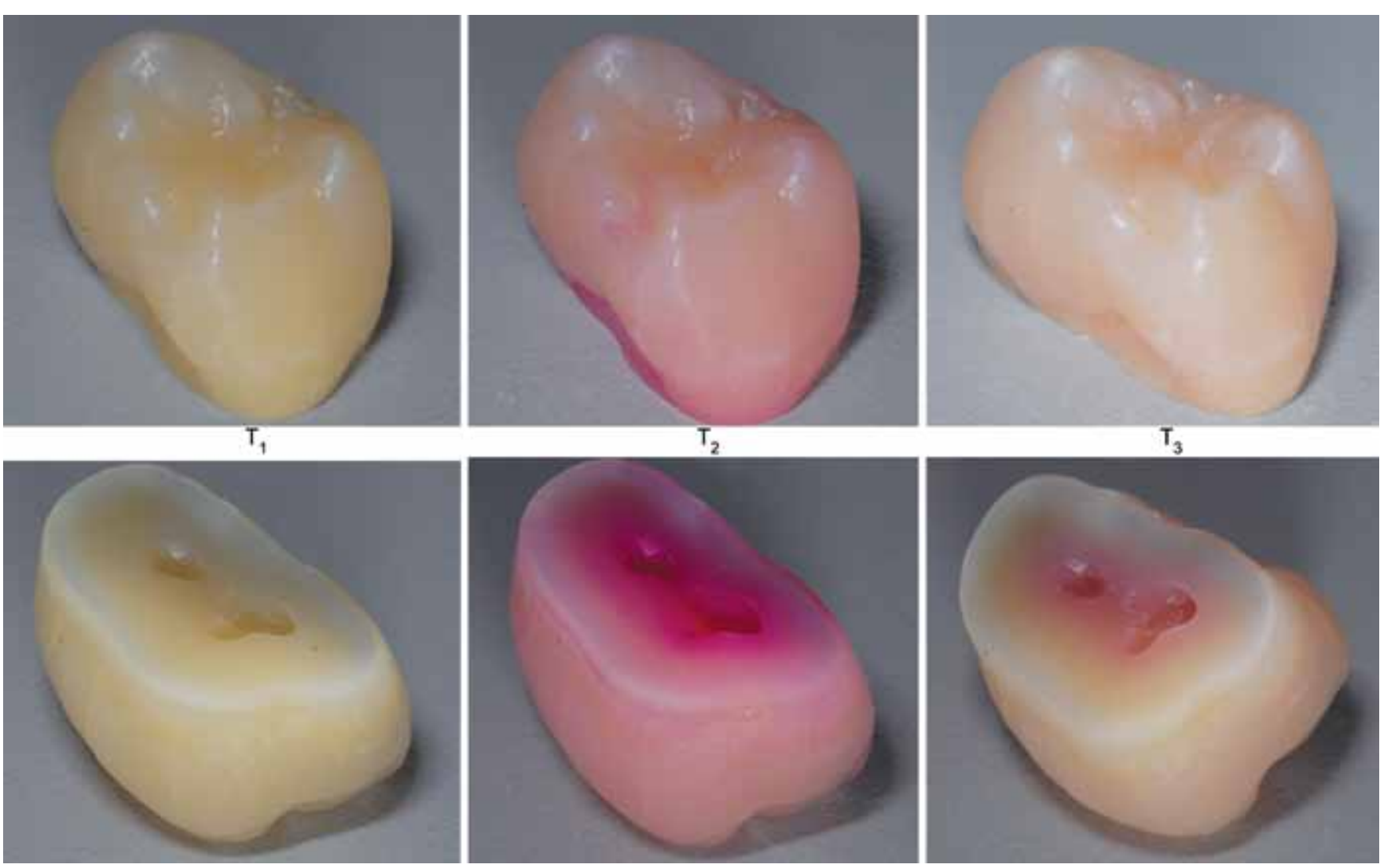

Fig. 2: Representative images of teeth at times $T_{1}, T_{2}$ and $T_{3}$

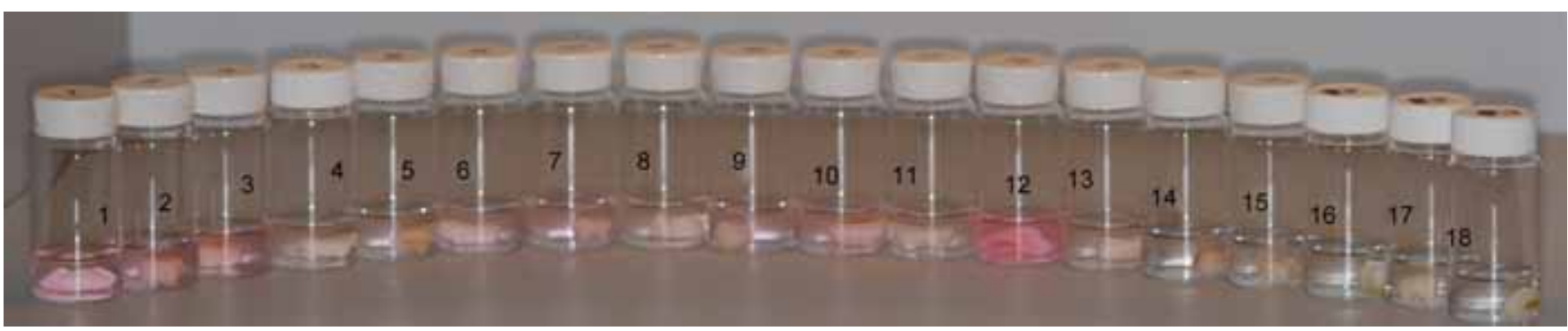

Fig. 3: Image of rinse solutions after 8 hours

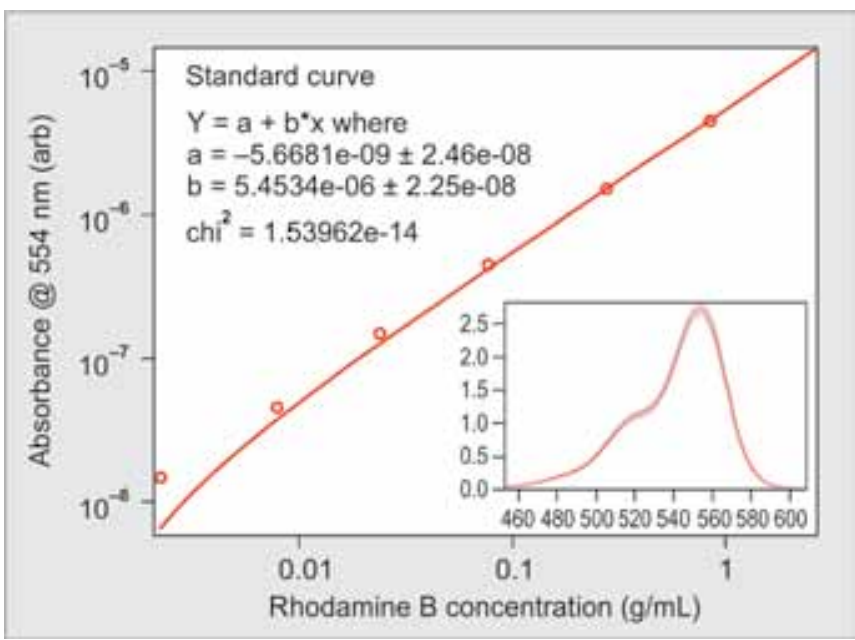

Graph 1: Standard absorption curve for Rhodamine B with an inset showing the Rhodamine $B$ absorbance spectrum
These changes are easily explained by the large absorption peak of Rhodamine B at $550 \mathrm{~nm}$, and significant absorption from 490 through $580 \mathrm{~nm}$ (Graph 1 inset). Since green is from about 520 to $550 \mathrm{~nm}$ while red is 600 to $700 \mathrm{~nm}$, it is quite clear that the value of (a) should have a significant positive shift. Also since blue is from about 450 to $500 \mathrm{~nm}$ while yellow is from about 550 to $600 \mathrm{~nm}$ and also the strongest absorption is from 530 to $570 \mathrm{~nm}$, it is understandable that there would be a slight negative shift in (b).

From the intermediate spectrophotometer readings at $\mathrm{T}_{2}$ a few additional insights were obtained. First, it was clear that teeth had more Rhodamine B dye immediately following staining, and thus all the color changes were exaggerated. However, it appears that there was about a 50\% rebound after rinsing for the (L), (h) and (a) parameters while there was 
a 70\% rebound in the (C) and (b). Again the stronger effect in the red/green balance is to be expected. We also observed a larger error from $T_{2}$ to $T_{3}$. This error likely indicates that there was a large spread in the dye retention from tooth to tooth at $\mathrm{T}_{2}$ perhaps due to natural variations, such as porosity.

\section{Measurement of Dye Retention}

The retained dye in a given tooth can be measured by taking the difference between an optical spectrum of the staining solution prior to staining and another spectrum after staining. Then by using a standard absorption curve (see Graph 1), the concentration of dye in each rinse solution together with the known volume of the solution can be used to calculate the total amount of dye in the solution. The spectrums used to calculate the retained dye are shown in Graph 2. As can be seen in the plots, the pre- and poststain spectrums plotted against the left axis are very similar, but the poststain spectrum has a measurably lower absorbance. The difference between the pre- and poststain solutions is plotted against the right axis along with the standard deviation of the poststain spectrums. The poststain spectra were averaged and then subtracted from the prestain solution to obtain the pre-/poststain difference shown in the graph. The standard deviation of the prestain solutions was negligible and is not shown since the error in the calculations is dominated by the standard deviation of the poststain solutions. As can be seen from the plots, the error (poststain solution standard deviation) in measuring the solutions is about half of the difference measured between the pre- and poststain solutions. While this error is high, there are straightforward methods for reduction of the errors in future experiments, and in the current analysis, the error may be magnified somewhat by the fact that the difference (blue dashed line in Graph 2) actually appears to be somewhat higher to the right of the peak at $553 \mathrm{~nm}$ (indicated by the solid vertical black line).

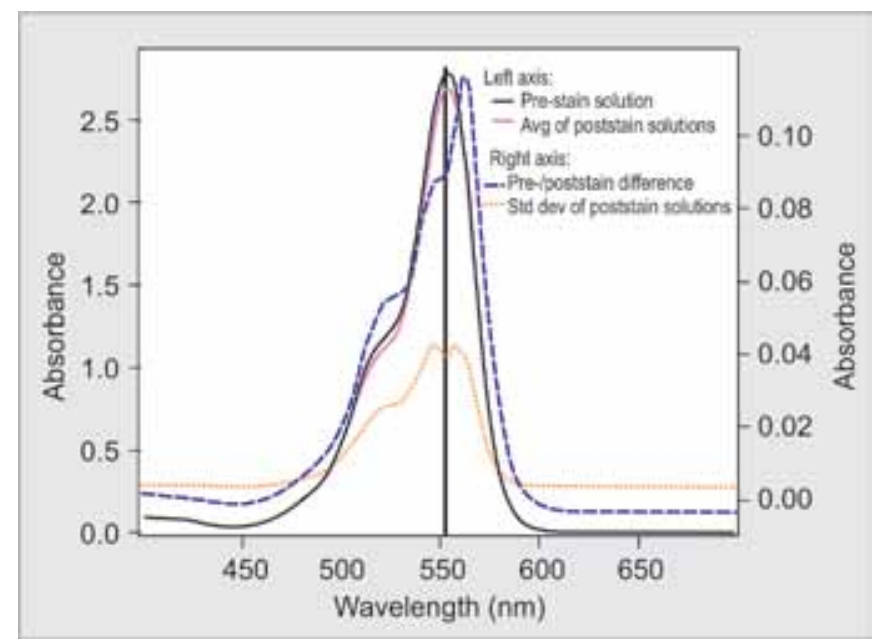

Graph 2: Absorbance spectrums for the rhodamine B solutions before and after staining the coronal sections
With the foregoing analysis and calculations, we were able to measure the absorbance of the pre- and poststain solutions at the $553 \mathrm{~nm}$ peak (indicated by vertical line in Graph 2) and, using the standard curve (see Graph 1), to calculate retention of stain molecules in the teeth to be $54 \pm 26 \mu \mathrm{g}$ of rhodamine B per gram of tooth.

\section{DISCUSSION}

Randomized controlled clinical trials provide the highest level of proof in evaluating the efficacy of tooth whitening. However, it is useful to develop laboratory models to evaluate various whitening materials and techniques that provide standardized and reproducible results. Sharif et al ${ }^{6}$ developed an extrinsic stain model with the use of tea and chlorhexidine. Pretty et $\mathrm{al}^{7}$ employed a stain model using a combination of human saliva, chlorhexidine and tea. Sulieman et $\mathrm{al}^{8}$ developed a reproducible intrinsic discoloration model based on tea. While previous studies used common comestible stain solutions, our study was based on Rhodamine B, which is a water-soluble molecule with a molar mass of $479 \mathrm{~g} / \mathrm{mol}$. The main advantage of Rhodamine B is its simple spectral signature enabling a robust spectral analysis. A confocal laser microscopy study showed that Rhodamine B when applied to the external surface of the tooth readily penetrated into the pulp via the interprismatic spaces in the enamel and dentinal tubules in the dentin supporting the validity of the use of Rhodamine B in our chromophore infiltration model. ${ }^{9}$

While the initial findings of this model show that dye retention in the teeth can be directly measured, the large standard deviation in retention spectra restricts the conclusions that can be drawn. However, it will be straightforward to reduce the errors associated with this method which come mainly from the fact that the amount of dye measured is small with respect to the stain solution volume used. To get smaller deviations, one must simply decrease the stain solution volume relative to the tooth volume. This reduction in stain solution volume may make it necessary to account for the porous volume in the teeth, but preliminary tests have been performed to show the porous volume can be easily measured to better than $10 \%$ of its actual value.

Based on the results of this study our null hypothesis was accepted. The use of solution density and optical absorption spectrums enabled the calculation of dye mass in extracted third molars after artificial chromophore infiltration. The ability to identify the mass of dye retained in the tooth provides standardized baseline parameters prior to tooth whitening. Tooth whitening efficacy can be directly measured by the change in mass of dye after the whitening procedure. This model may also be applied to different dye 
molecules to evaluate the whitening potential based on stain spectrum, molecular weight, chemical structure and other parameters.

\section{CONCLUSION}

Using solution density and optical absorption spectrums, we were able to directly calculate the mass of dye retained in extracted third molars.

\section{REFERENCES}

1. Haywood VB. History, safety and effectiveness of current bleaching techniques and applications of the nightguard vital bleaching technique. Quintessence Int 1992;23(7):471-488.

2. Albers H. Lightening natural teeth. ADEPT Report 1991;2(1): 1-24.

3. Ushigome T, Takemoto S, Hattori M, Yoshinari M, Kawada E, Oda Y. Influence of peroxide treatment on bovine enamel surface — cross-sectional analysis. Dent Mater J 2009;28(3): 315-323.

4. Markovic L, Fotouhi K, Lorenz H, Jordan RA, Gaengler P, Zimmer S. Effects of bleaching agents on human enamel light reflectance. Oper Dent 2010;35(4):405-411.

5. Ito Y, Momoi Y. Bleaching using $30 \%$ hydrogen peroxide and sodium hydrogen carbonate. Dent Mater J 2011;30(2):193-198.

6. Sharif N, MacDonald E, Hughes J, Newcombe RG, Addy M. The chemical stain removal properties of 'whitening' toothpaste products: studies in vitro. Br Dent J 2000;188:620-624.
7. Pretty IA, Edgar WM, Higham SM. The use of QLF to quantify in vitro whitening in a product testing model. Br Dent J 2001; 191:566-569.

8. Sulieman M, Addy M, Rees JS. Development and evaluation of a method in vitro to study the effectiveness of tooth bleaching. J Dent 2003;31:415-422.

9. Kwon SR, Wertz PW, Li Y, Chan DCN. Penetration pattern of Rhodamine dyes into enamel and dentin: confocal laser microscopy observation. International Journal of Cosmetic Science 2012;34:97-101.

\section{ABOUT THE AUTHORS}

\section{R Steven Kurtir Jr (Corresponding Author)}

Associate Professor and Director, Biomaterials and Photonics Research Laboratory, Loma Linda University School of Dentistry, 11092 Anderson St. Loma Linda, CA 92350, USA, Phone: (909) 558-0656 Fax: (909) 558-0270, e-mail: skurti@llu.edu

\section{Berj Der Boghossian}

Research Volunteer, Department of Restorative Dentistry, Center for Dental Research, Loma Linda University School of Dentistry, 11092 Anderson St. Loma Linda, CA 92350, USA

\section{So Ran Kwon}

Associate Professor, Department of Operative Dentistry, University of Iowa College of Dentistry, Iowa city, IA, USA 